Mots. Les langages du politique

\title{
Une médiatisation improbable ? Fermeture d'une usine rurale et intérêts journalistiques
}

An improbable media coverage? Shutdown of a rural factory and journalistic interests

¿Una mediatización improbable? Cierre de una fábrica rural e intereses

periodisticos

Olivier Baisnée et Frédéric Nicolas

\section{OpenEdition}

\section{Journals}

Édition électronique

URL : http://journals.openedition.org/mots/22976

DOI : $10.4000 /$ mots.22976

ISSN : 1960-6001

Éditeur

ENS Éditions

\section{Édition imprimée}

Date de publication : 1 novembre 2017

Pagination : 89-107

ISSN : 0243-6450

Référence électronique

Olivier Baisnée et Frédéric Nicolas, « Une médiatisation improbable? Fermeture d'une usine rurale et intérêts journalistiques », Mots. Les langages du politique [En ligne], 115 | 2017, mis en ligne le 21 novembre 2019, consulté le 05 janvier 2021. URL : http://journals.openedition.org/mots/22976 ; DOI https://doi.org/10.4000/mots.22976 


\section{Une médiatisation improbable? Fermeture d'une usine rurale et intérêts journalistiques}

Le 23 octobre 2008, la direction américaine du groupe Molex, fabricant de connectique, annonce la fermeture du site de Villemur-sur-Tarn et le licenciement de 282 salariés. Si cette déclaration est concomitante avec la crise qui frappe les marchés financiers en cet automne 2008, elle ne lui est bien entendu pas imputée : la fermeture de cette usine achetée en 2004 avait en effet été préparée en secret depuis longtemps. Avec la complicité de certains cadres intermédiaires locaux, une copie d'une partie de l'outil industriel avait été réalisée afin de ne pas entraîner de rupture d'approvisionnement des constructeurs automobiles pour lesquels travaille Molex. Véritable palimpseste symbolique, la lutte de ces salariés, pendant plus d'un an, va devenir dans le discours journalistique, syndical (Bernard Thibault parlant à son propos de «cas d'école») et politique (tout le spectre politique la mobilisant en exemple) un des symboles de la crise que traverse la France et de la désindustrialisation de son économie.

Cette mobilisation qui dure près d'un an ${ }^{1}$, et dont le versant judiciaire n'est toujours pas achevé, va compter au rang des conflits les plus médiatisés parmi les milliers de plans de sauvegarde de l'emploi annoncés tous les ans². Ces 282 ouvriers provinciaux, ruraux et âgés ${ }^{3}$, travaillant pour une société inconnue (sous-traitante de l'automobile) et fabriquant un produit n'évoquant rien (des connecteurs) vont pourtant, malgré ces propriétés qui en faisaient de piètres candidats à l'accès aux médias nationaux (y compris audiovisuels), être constitués en symbole et bénéficier d'une couverture journalistique difficilement imaginable, sauf à tomber dans le piège téléologique qui menace toute analyse

1. Le 15 septembre 2009 les salariés autorisent leurs représentants à se prononcer sur le Plan de sauvegarde de l'emploi.

2. 1048 en 2008, 2242 en 2009.

3. En septembre 2009, la moyenne d'âge était de 46,3 ans. Près de $40 \%$ des salariés avaient plus de 50 ans. L'ancienneté moyenne des ouvriers était de 22,7 années et près d'un tiers avait plus de 30 ans d'ancienneté.

IEP de Toulouse, Laboratoire des sciences sociales du politique olivier.baisnee@sciencespo-toulouse.fr Centre d'économie et de sociologie appliquées à l'agriculture et aux espaces ruraux (INRA-AgroSup Dijon), Laboratoire des sciences sociales du politique (IEP de Toulouse) frederic.nicolas@sciencespo-toulouse.fr 
des phénomènes médiatisés. La médiatisation doit en effet toujours demeurer quelque chose à expliquer tant elle constitue l'exception (moins de $5 \%$ des événements protestataires) et non la règle en matière d'action collective (Fillieule, 1996 et 2007). Reste donc un questionnement stimulant à défaut d'être facile à résoudre : comment expliquer la « réussite » médiatique de ce conflit? Cette médiatisation constitue, en effet, une «réussite »4 tant en termes d'attention journalistique que de cadrages 5 ou, mieux, d'ordres de discours ou de "schèmes d'interprétation» (Goffman, 1974) appliqués à cette mobilisation. Les salariés reconnaissent en effet avoir été «bien traités» par les journalistes et avoir réussi à «faire passer leur message». Par ailleurs, ce site industriel, et plus largement cette entreprise, n'existaient nullement médiatiquement : la quasi-intégralité des 1855 articles publiés entre 2000 et 2014 évoquant Molex l'ont été à l'occasion de ce conflit 6 . Enfin, ce conflit qui concerne un nombre limité de salariés et se déroule dans le Sud-Ouest de la France, va accéder au statut d'événement couvert par les journaux et les médias audiovisuels nationaux et non uniquement régionaux 7 [voir graphe 2]. Pour autant les bénéfices de cette médiatisation du conflit ne doivent bien entendu pas être surestimés. Comme le dira, avec une lucidité désarmante, un des anciens salariés le plus au contact des journalistes :

[Les journalistes] nous ont souvent dit qu'on était rentrés dans l'histoire, et je leur ai souvent répondu que ça me faisait une belle jambe [Rires].

Cette analyse d'un corpus de presse ne vise pas, comme le font les études en termes de frame analysis, à saisir comment la mise en symbole et en sens de la situation parvint à mobiliser les salariés, mais à tenter de saisir comment furent produites les interprétations de ce conflit du travail. Il convient dès lors d'analyser ce que la presse française a donné à voir médiatiquement de ce conflit et les ressorts de cette attention journalistique. Cela est rendu possible par une enquête collective qui associe neuf enseignants-chercheurs, doctorants et post-doctorants (O. Baisnée, A. Bory, B. Crunel, É. Darras, C. Frau, A. Oeser, A. Rouger, Y. Selponi) et qui a bénéficié d'un financement ANR ${ }^{\text {. Elle }}$

4. Qualificatif piégé pour les sciences sociales mais on peut suspendre l'objection en relevant que, pour les acteurs concernés, elle fut effectivement « réussie» (voir infra).

5. «Les cadres de l'action collective sont [...] des ensembles de croyances et de significations, orientés vers l'action, qui inspirent et légitiment les activités et les campagnes des organisations de mouvement social» (Benford et al., 2012, p. 224).

6. Entre juin 2000 et début octobre 2008 , seulement quatorze articles mentionnant Molex sont répertoriés. Pour le seul mois d'octobre 2008, pas moins de vingt-cinq articles sont consacrés à l'usine.

7. Choisir d'inclure la presse quotidienne régionale et la presse quotidienne nationale dans le corpus nous a permis précisément d'objectiver cet aspect de la réussite médiatique de la mobilisation.

8. Un ouvrage tiré de cette enquête est paru en 2017 : Collectif du 9 août, Quand ils ont fermé l'usine (2017). Une partie des éléments avancés ici sont tirés du chapitre 5 de cet ouvrage. Conformément aux principes adoptés par l'équipe certains enquêtés et noms propres ont été anonymisés. 
repose sur une centaine d'entretiens avec d'anciens salariés de l'usine, mais aussi avec des acteurs qui, à un titre ou à un autre (journalistes, avocats, experts, responsables politiques, dirigeants étasuniens), ont joué un rôle dans l'histoire sociale et locale de cette fermeture d'usine et de la lutte de ses salariés. La recherche se fonde également sur de nombreuses observations faites dans des circonstances variées, qu'elles aient à voir avec la lutte (audiences au tribunal, cortèges lors de manifestations, événements musicaux et plus largement culturels liés à la lutte, assemblées générales de l'association des anciens salariés) ou qu'elles procèdent du dispositif de l'enquête lui-même (enquête collective effectuée par séjours réguliers d'une semaine, deux à trois fois par an) : en travaillant régulièrement en quasi-immersion au sein du local de l'association Solidarité Molex, situé (jusque récemment) en centre-ville, en bénéficiant de relations d'amitié, nous avons eu accès aux espaces privés, au quotidien de certains de ces anciens salariés bien au-delà de leur collectif. À l'enquête collective s'est finalement agrégée la construction d'un corpus de presse exhaustif autour de la fermeture de l'usine Molex, afin de repérer les prises de position dans le conflit et d'étayer ainsi des observations empiriques. Ce corpus de presse de 1855 articles a fait l'objet d'un traitement statistique à l'aide du logiciel libre Iramuteq, développé au sein du Laboratoire d'études et de recherches appliquées en sciences sociales (LERASS, Université Paul Sabatier, Toulouse) 9 .

\section{Une médiatisation réussie}

L'analyse quantitative du corpus permet à bien des égards de «durcir» des hypothèses issues de l'enquête ethnographique et des entretiens avec les anciens salariés - où était très souvent évoqué, parfois «dossiers de presse» à l'appui, le rôle des médias et des journalistes - comme avec les journalistes ayant couvert la mobilisation. Si l'analyse de cadres repose la plupart du temps sur des analyses qualitatives, le fait de regrouper des communautés de contexte dans des classes à l'aide d'Iramuteq nous a permis d'objectiver ce qui n’était au départ qu'un ressenti d'ethnographes ${ }^{10}$, à savoir que la médiatisation de la fermeture de l'usine Molex était réussie, tant du point de vue du nombre d'articles (analyse statistique simple) que des cadrages proposés (analyse par classification ${ }^{11}$ ). Les processus de cadrage sont donc entendus ici au sens quasi-photographique de ce qui se trouve associé dans le discours

9. L'analyse lexicométrique a été rendue possible par un financement du Labex SMS.

10. On peut d'ailleurs rappeler utilement que l'analyse de cadres, souvent comprise, par un effet de traduction sélective, comme avant tout théorique et fondée en données chiffrées, est aussi issue d'une tradition ethnographique, ce que la trajectoire de David Snow permet de mieux saisir (Jouanneau, 2012).

11. Iramuteq reproduit la méthode de classification décrite par M. Reinert (1983). 
journalistique (ce qui est donc « dans le cadre ») c'est-à-dire inséparablement de ce qui n'est pas mis en relation et reste, dès lors, « hors champ».

\section{La pesée globale : une médiatisation continue}

D’un point de vue strictement quantitatif, 1855 articles mentionnant « Molex» ont été publiés dans l'ensemble de la presse écrite française entre 2000 et fin $2014^{12}$. La presse quotidienne régionale (PQR) représente 41,9\% du corpus avec 777 articles mentionnant Molex (dont 531 de La Dépêche, soit 68,3\% de la PQR), contre 32,6 \% (605 articles) pour la presse quotidienne nationale (PQN) - dont L'Humanité (228), Le Parisien (110), Le Monde (82), Libération (82), Le Figaro $(65)^{13}$. En nous en tenant à un strict comptage, nous pouvons faire trois constats. Tout d'abord, la médiatisation de la mobilisation apparaît encore plus réussie si nous la comparons à la couverture médiatique reçue par la mobilisation autour de la fermeture d'une autre usine de la région à la même période [voir Graphe 1] : en effet, la mobilisation autour de la fermeture de l'usine de composants microélectroniques Freescale (Toulouse) est loin de recevoir une couverture médiatique aussi importante que celle accordée aux Molex (tout type de presse confondu) ${ }^{14}$.

Ensuite, de l'annonce de la fermeture de l'usine (23 octobre 2008) au jugement des prud'hommes (27 mars 2014), la presse écrite porte une attention continue à la mobilisation, surtout d'octobre 2008 à décembre 2010 avec quatre pics d'attention correspondant à l'annonce de la fermeture et à l'opération «ville morte» qui suit, à la retenue de deux cadres de l'usine, à la décision des employés de donner mandat à leurs responsables pour discuter du plan social puis à la décision des employeurs de ne plus le financer tant que les plaintes aux prud'hommes n'auront pas été abandonnées [voir Graphe 2].

Enfin, malgré les caractéristiques de l'entreprise et de ses employés, la médiatisation de la mobilisation concerne autant la $P Q R$ que la $P Q N$, notamment à partir du moment de la retenue des cadres et de la décision d'amorcer le processus du plan social. L'événement de la fermeture et ses suites ont donc une visibilité nationale à laquelle n'accèdent pas toutes les fermetures.

12. Le corpus a été constitué en consultant les plateformes Europresse et Factiva, ainsi que les sites Internet des journaux Le Monde et La Dépêche du Midi. Le mot-clé utilisé a été «Molex» dans l'«ensemble de l'article». À l'analyse qualitative et partielle de la presse écrite pendant l'enquête ethnographique est donc venue s'agréger l'analyse statistique de l'ensemble du corpus sur laquelle repose principalement cet article.

13. Les dépêches d'agence sont au nombre de 446 , soit $24 \%$ du corpus. Les articles de la presse hebdomadaire nationale (PHN) sont au nombre de 27 , soit 1,5\% du corpus.

14. Sur l'échantillon composé de titres de PQN (Le Monde, Libération, Le Figaro, Le Parisien, L'Humanité) et d'un titre de la PQR (La Dépêche du Midi), Molex fait l'objet de 1087 articles, soit presque deux fois plus que Freescale (592). 


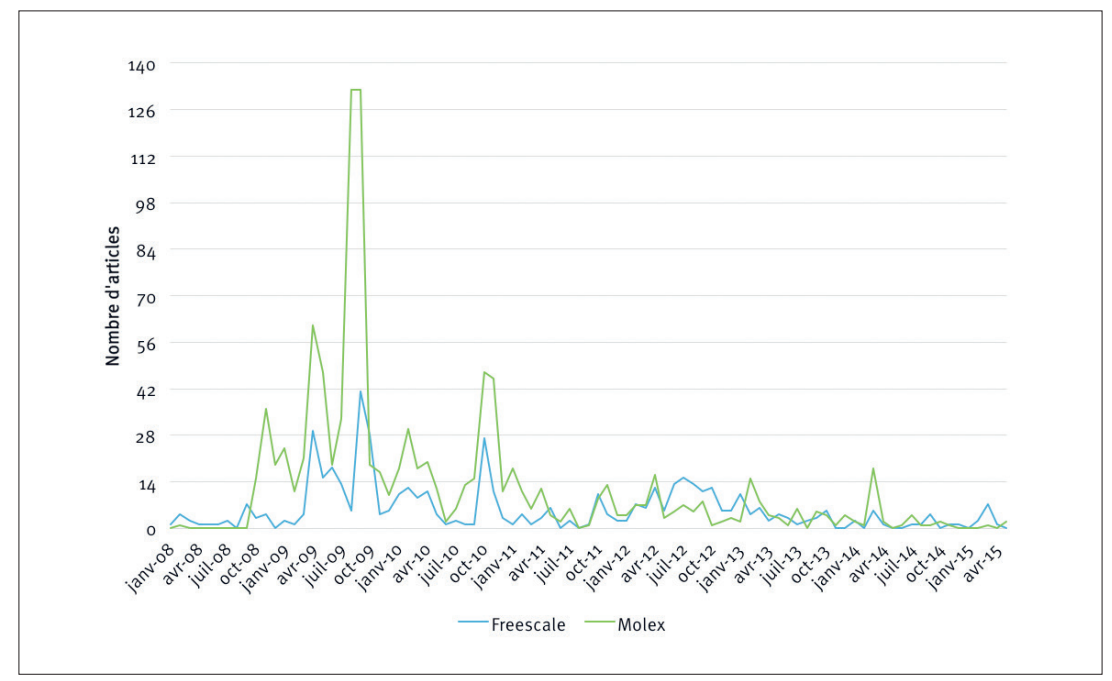

Graphe ${ }^{0}{ }^{1}$. Comparaison Freescale-Molex sur un échantillon de titres de la presse écrite

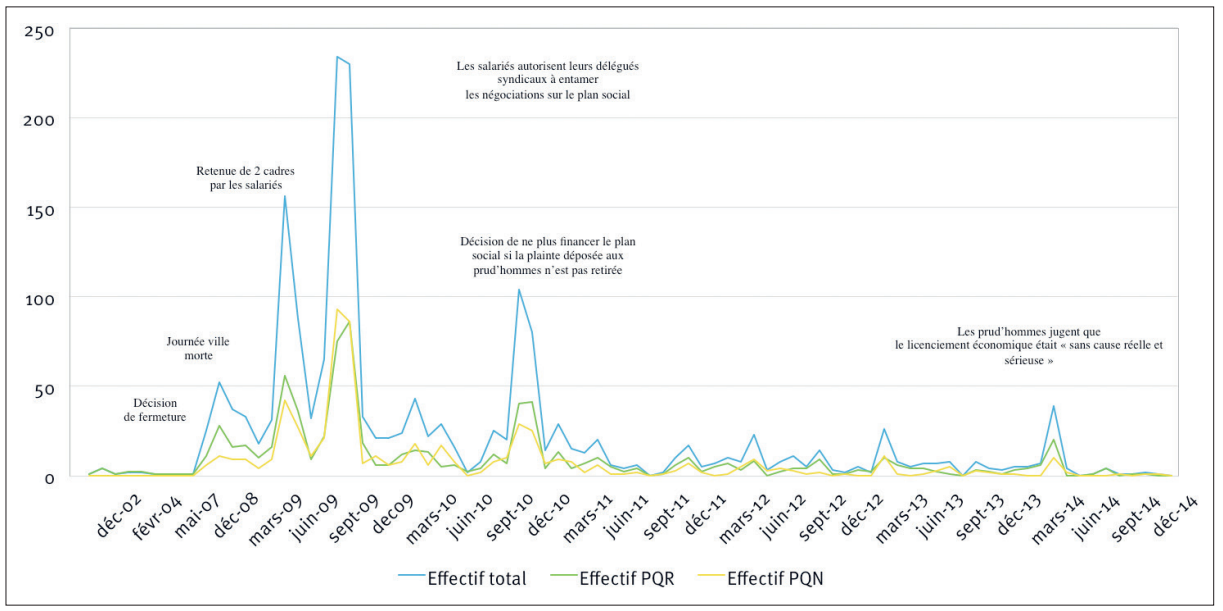

Graphe n². Chronologie de la mobilisation Molex et de sa couverture médiatique 


\section{La mobilisation des Molex : un «cas d'école»}

La réussite de la médiatisation de la mobilisation se mesure également à l'aune des cadrages retenus par la presse. Sur les 1855 articles qui composent le corpus, cinq cadrages peuvent être isolés, puis regroupés en deux groupes d'égale importance.

Il y a d'abord ce qui relève des cadrages généraux ou de montée en généralité. Ils sont de deux ordres. Le premier (30,5\%) (classe 1 sur le dendrogramme $\mathrm{n}^{0}{ }_{1}^{1}$ ) consiste à faire de Molex un cas emblématique ou exemplaire, un symbole des conséquences de la crise sur l'emploi ${ }^{15}$ : la perspective adoptée dans les articles privilégiant ce cadrage est celle des chiffres du chômage et des politiques mises en place pour les faire baisser. Molex n'apparaît pas dans le titre; il s'agit d'un cadrage davantage adopté par la presse nationale hebdomadaire (Le Point) ${ }^{16}$ et quotidienne (L'Humanité notamment) ; enfin, c'est un cadrage qui concerne moins les périodes importantes du conflit que les échéances électorales ou en termes de politiques publiques (annonce des chiffres du chômage, campagne électorale, etc.).

Toulouse, correspondance. Le résultat du vote est clair et net : par 67 voix pour et o contre, le conseil régional de Midi-Pyrénées vient d'adopter un vœu qui appelle à une loi interdisant les licenciements boursiers [...]. Le texte fait référence à des entreprises présentes en Midi-Pyrénées : « Après Molex, Thales et Freescale, c'est maintenant Sanofi, dont les actionnaires ont perçu 3,5 milliards d'euros de dividendes en 2011, qui procède à des suppressions d'emplois à visées boursières ». Le licenciement boursier, selon la définition donnée par le texte, est « le résultat d'une course cynique au rendement financier» dans des entreprises pourtant en bonne santé économique. (L'Humanité, 20 novembre 2012)

À l'inverse, le second cadrage général (23,2 \%) (classe 5 sur le dendrogramme $n^{0}{ }_{1}$ ) est plutôt caractéristique des moments-clés de la mobilisation dans la rue et aux prud'hommes (2009 et 2014). Il concerne les enjeux d'appropriation de la mobilisation au sein du champ politique et des organisations syndicales, et entre organisations politiques et syndicales. Molex est alors un prétexte pour soulever d'autres enjeux (le nom de l'usine n'apparaît pas dans le titre). Contrairement au cadrage précédent, il s'agit d'un cadrage plutôt caractéristique de la presse régionale (La Dépêche du Midi, mais également Midi Libre et Sud-Ouest). Les acteurs de la vie politique locale (présidents de la région, du conseil départemental et de la chambre de commerce, maire de Villemur-surTarn, maire de Toulouse) y sont présentés comme des intercesseurs auprès de l'exécutif (le président Sarkozy et son ministre de l'industrie Christian Estrosi)

15. Et ce alors même que l'annonce de la fermeture n'est en rien liée à la crise des marchés financiers. 16. Nous rappelons toutefois que seulement 27 articles de la PHN figurent dans notre corpus. 
et de certains représentants du PS (Martine Aubry, Ségolène Royal, etc.) tandis que les visites du site par les leaders syndicaux (celle de Bernard Thibault notamment) et de l'extrême gauche (celle d'Olivier Besancenot notamment) font l'objet de descriptions factuelles, accompagnées de verbatim.

Les deux premiers cadrages isolés attestent ainsi de la relative réussite de la médiatisation dans la mesure où la fermeture de l'usine Molex semble constituer un enjeu de visibilité pour les acteurs publics, politiques et syndicaux, à l'échelle locale et nationale (assez en tout cas pour que des prises de position soient énoncées et que des visites soient organisées en présence de la presse et à destination de la presse). Cela relève d'une médiatisation sur laquelle «les Molex» n'ont à peu près aucune prise, ou en tout cas pas de manière directe.

Une seconde catégorie de cadrages émerge autour des mobilisations proprement dites. Ils sont de trois ordres principalement. Le premier $(18,3 \%)$ (classe 3 sur le dendrogramme $n^{0} 1$ ) concerne la mobilisation dans la rue pour la défense de l'emploi et de l'outil de travail : il s'agit d'une part d'un cadrage présent tout au long de la mobilisation, y compris lorsque la mobilisation passe dans sa phase judiciaire, après 2009; et d'autre part d'un cadrage spécifique aux agences et dans une moindre mesure aux journaux Le Monde et Le Parisien.

Le second $\left(14,3 \%\right.$ ) (classe 2 sur le dendrogramme $\mathrm{n}^{0}{ }^{1}$ ) concerne les négociations tripartites autour de la fermeture et de la reprise de l'usine : Molex constitue le sujet principal des articles adoptant ce cadrage; il s'agit d'un cadrage davantage employé par les agences que par les autres types de presse écrite; enfin, sans réelle surprise, il s'agit d'un cadrage mobilisé sur une courte période de temps correspondant précisément au moment des négociations (août à octobre 2009).

Haute-Garonne: B.Thibault plaidera à l'Elysée le cas des salariés de Molex. [...] "Vous êtes un peu un cas d'école, dans une de ces entreprises qui dégagent des bénéfices mais qui mettent la crise économique en avant pour tout justifier, y compris des licenciements dont rien ne prouve le bien-fondé», a déclaré le leader syndical devant plus d'une centaine de salariés de Molex réunis devant le portail de l'entreprise. (Dépêche AFP, 30 octobre 2008)

Le troisième cadrage $(13,7 \%)$ (classe 4 sur le dendrogramme nº 1 ) concerne la mobilisation du droit dans le conflit qui oppose les employés à leur employeur. L'enjeu est de disqualifier les motifs de fermeture et d'obtenir réparation auprès des Prud'hommes : il s'agit d'une part d'un cadrage ponctuel correspondant au feuilleton judiciaire, c'est-à-dire à des événements/étapes amenant jusqu'au dénouement en mars 2014 (reconnaissance que le licenciement était sans cause réelle et sérieuse); et d'autre part d'un cadrage qui semble caractéristique des agences et dans une moindre mesure du journal Le Monde.

Les Molex attendent la condamnation jeudi de leurs «licenciements illicites». Les anciens salariés de l'usine française de connectique automobile du groupe 


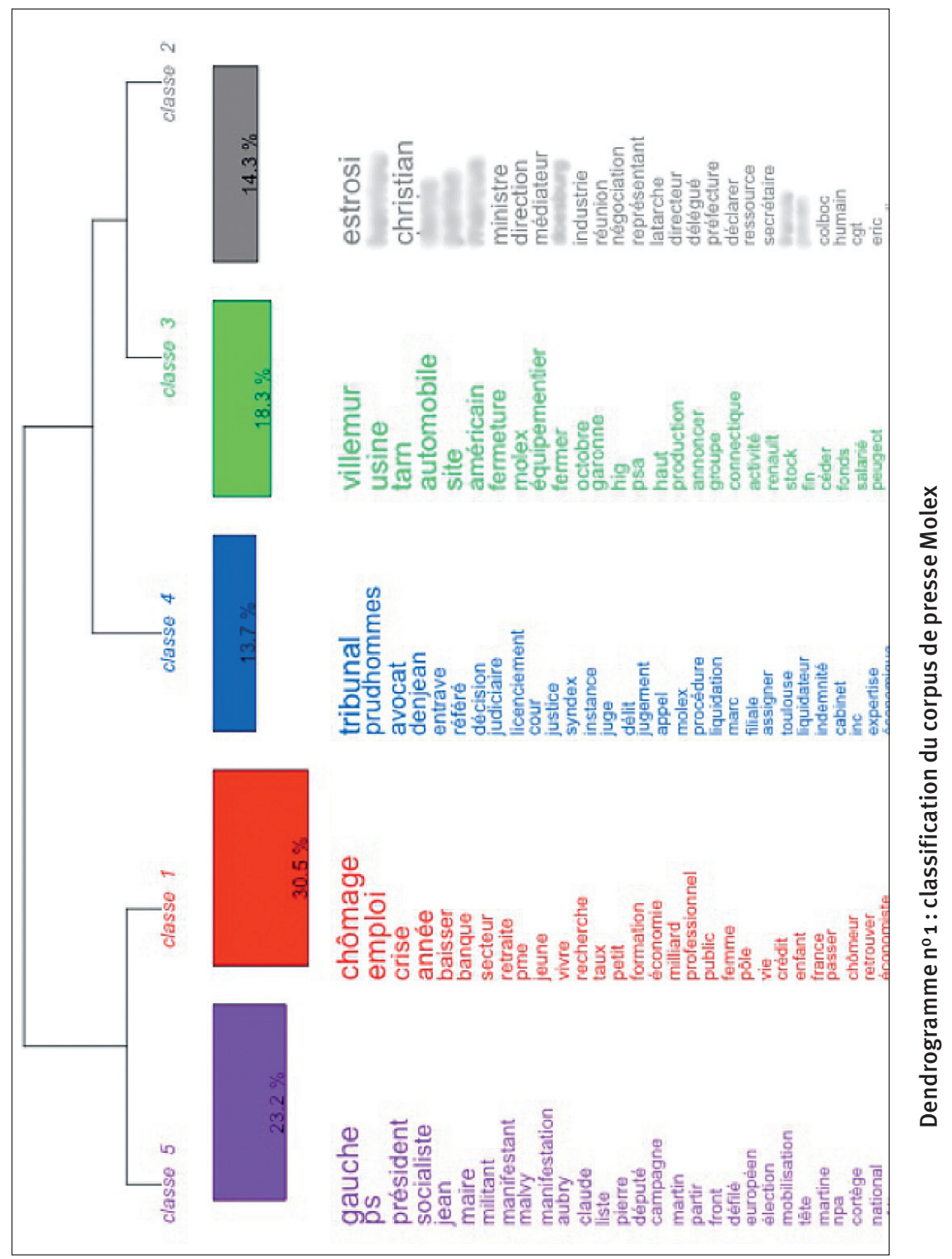




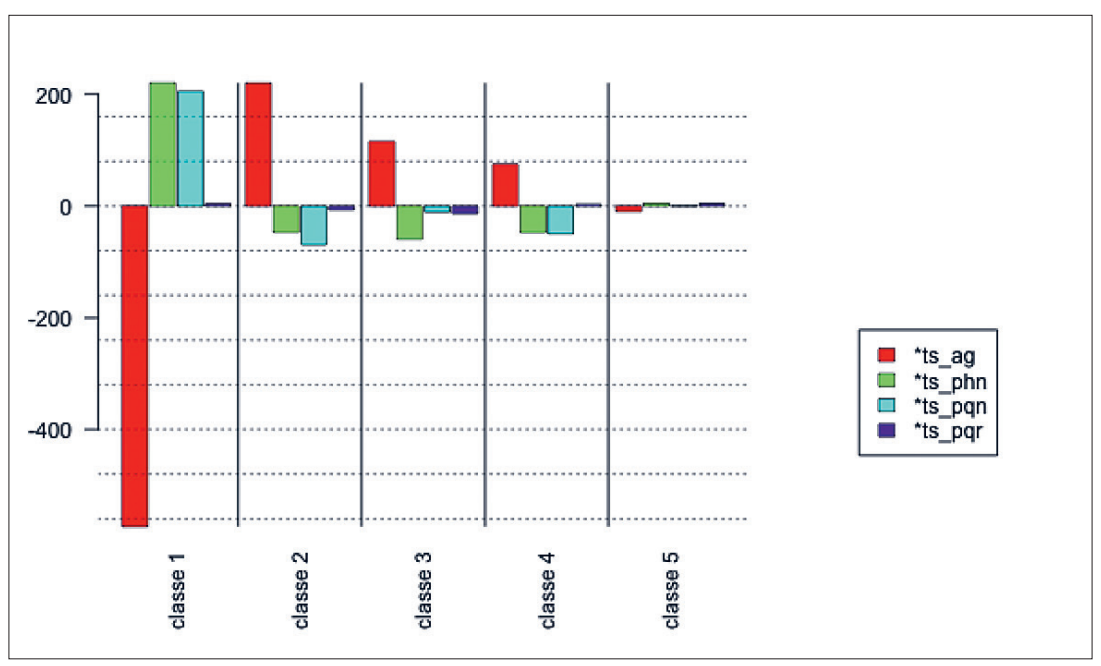

Graphe $n^{0} 3 \cdot \chi^{2}$ par modalités de la variable «Type de sources» (Agences de presse, $\mathrm{PHN}, \mathrm{PQN}, \mathrm{PQR}$ )

américain Molex seront nombreux jeudi au tribunal des prud'hommes de Toulouse avec l'espoir d'une dernière victoire juridique : la condamnation de leur ancien patron pour licenciements illégitimes. (Dépêche AFP, 25 mars 2014)

Cette deuxième catégorie de cadrages autour des mobilisations permet de souligner que la presse a couvert l'ensemble du conflit (de 2008 à 2014) dans ses volets social, politique et judiciaire. Cette première analyse du corpus de presse permet ainsi de souligner que Molex est un enjeu visible en même temps qu'un enjeu de visibilité pour des acteurs et groupes d'acteurs concernés directement (employés et employeur) ou indirectement (gouvernement et organisations politiques et syndicales) par la fermeture de l'usine.

Néanmoins, ce qui apparaît peut-être moins nettement, c'est la manière dont la PQR (et au sein de la PQR le journal lu par «les Molex»: La Dépêche du Midi) traite le sujet ${ }^{17}$ et les différences qui peuvent exister entre le traitement proposé par cette dernière et la PQN. Pour faire émerger ces différences, il a été nécessaire de constituer des sous-corpus par types de presse (PQN, $P Q R$, PHN, Agences de presse) ainsi qu'un sous-corpus PQR-PQN. Cela a permis de mieux comprendre la spécificité des cadrages de chaque type de presse écrite.

Le sous-corpus PQN se caractérise par des cadrages similaires au corpus général (négociations tripartites ${ }^{18}$, mobilisation du droit19, emploi/chômage

17. Voir graphe 3, où la PQR n'est statistiquement corrélée à aucune des classes de discours isolées.

18. Caractéristique de l'ensemble des titres de PQN.

19. Caractéristique du journal Le Monde. 


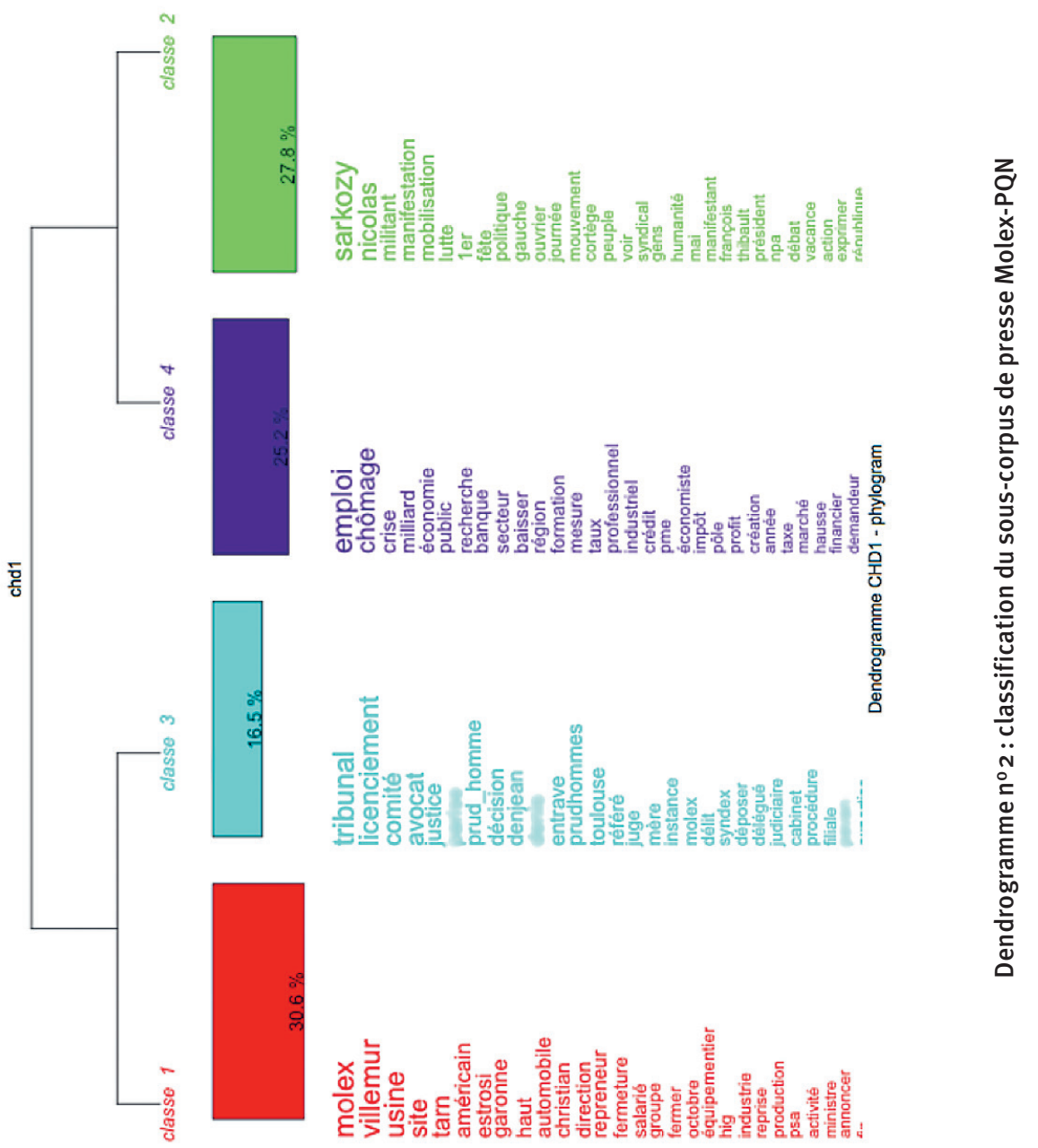




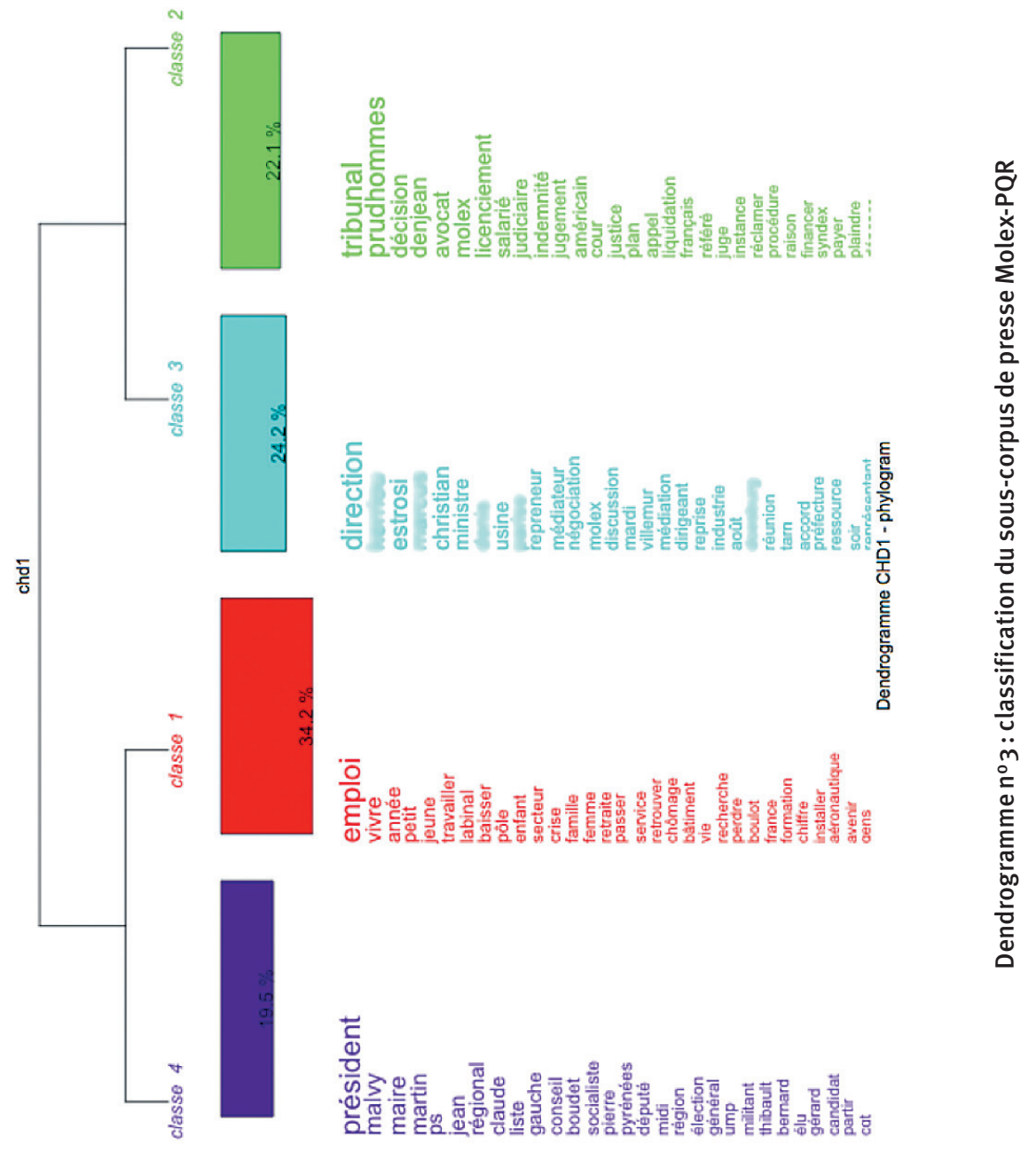




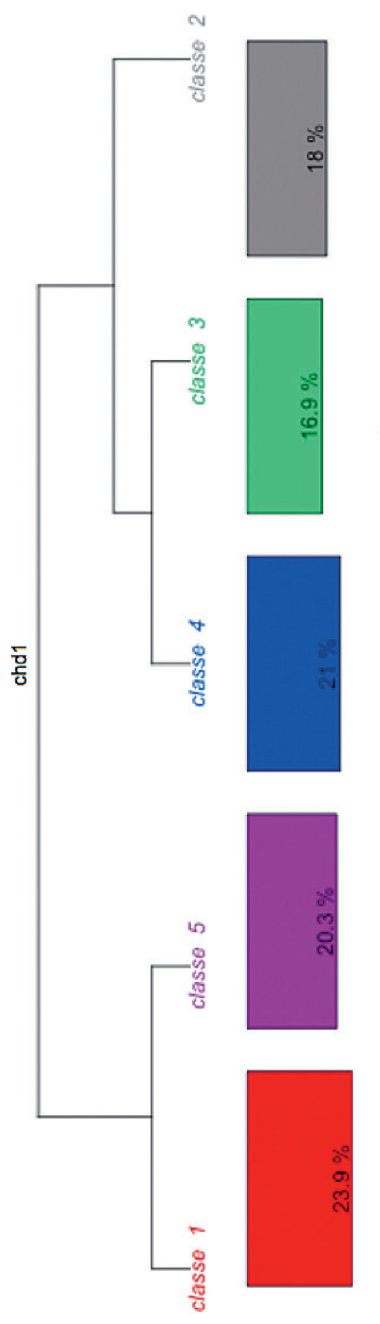

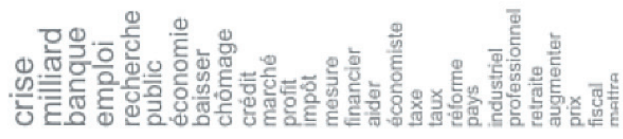

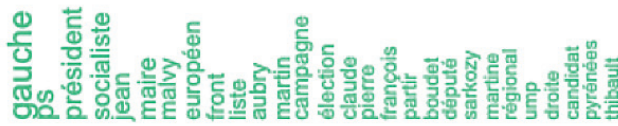

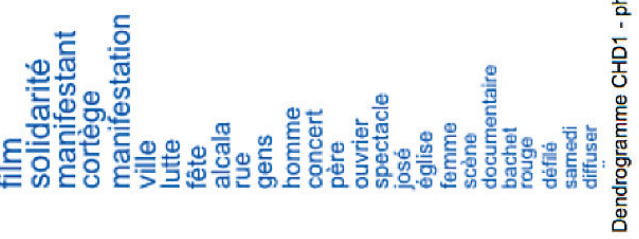


dans une perspective de politique publique ${ }^{20}$ ), mais également par un cadrage qui lui est propre : la fermeture de l'usine Molex est une occasion ("Molex» n'apparaît pas dans le titre) de parler des relations ouvertement conflictuelles entre le président Sarkozy et les organisations syndicales (dans le contexte de la «petite phrase » de Nicolas Sarkozy, prononcée en juillet 2008 : «Désormais, quand il y a une grève en France personne ne s'en aperçoit "). Il s'agit d'un cadrage qui est spécifique à la PQN de "gauche» (Libération et L'Humanité).

À l'inverse, le sous-corpus PQR fait émerger un cadrage singulier qui n'apparaît pas dans le corpus général : celui des conséquences de la fermeture sur la vie professionnelle et familiale des licenciés. La Dépêche est fortement corrélée à ce cadrage : les articles consacrés à cette thématique insistent sur la résilience des individus face à la crise, notamment à travers des portraits de reclassés ou de personnes (dans l'entreprise et hors-entreprise) mobilisées pour la sauvegarde de l'emploi ou « ayant envie de travailler». Ce type de couverture médiatique de la mobilisation participe ainsi à la construction d'une cause moralement irréprochable, dont il nous faut dans un second temps définir les contours, en nous intéressant à la perception de la médiatisation du conflit par «les Molex» (positivement perçue comme leur renvoyant une bonne image d'eux-mêmes).

Enfin, le sous-corpus PQR-PQN (c'est-à-dire excluant les articles des agences et de la presse hebdomadaire nationale, qui ont tendance à écraser le corpus général par de «l'événementiel » pour les premiers, et qui n'étaient pas réellement significatifs pour les seconds) permet de faire apparaître un cadrage qui n'apparaissait pas dans le corpus comprenant l'ensemble des articles consacrés à la mobilisation, et ainsi de préciser la spécificité du cadrage proposé par la PQR et La Dépêche en particulier. Il s'agit d'un cadrage autour de la mobilisation, envisagée dans son contexte local, et centré sur ce qu'elle occasionne en termes de sociabilités locales, festives et militantes.

Non seulement la fermeture de l'usine Molex ne passe pas inaperçue (contrairement à la majorité des fermetures d'usine) mais elle devient un enjeu de la compétition politique et de la construction des politiques publiques (localement et nationalement). Surtout, la médiatisation de la mobilisation contre la fermeture puis pour la condamnation de Molex ne fait pas l'objet d'un traitement négatif de la part de la presse, contrairement à d'autres mobilisations : celle de Continental-Clairoix (passée à la postérité pour le «saccage de la sous-préfecture de Compiègne») ou celle des salariés d'Air France contre un plan de « restructuration » visant 2900 emplois (entrée dans la mémoire collective comme «l'affaire de la chemise arrachée »). Fort de cette objectivation statistique, il devient dès lors nécessaire de comprendre, grâce à l'enquête ethnographique, les ressorts sociaux de cette médiatisation aussi réussie qu'improbable.

20. Caractéristique de L'Humanité.

Mots. Les langages du politique $\mathrm{n}^{\circ} 115$ novembre $2017 \bullet 101$ 


\section{Les ressorts d'une médiatisation « improbable»}

Si Toulouse accueille des correspondants de nombreux médias nationaux et un bureau de l'AFP, Villemur en est à la fois proche (une trentaine de kilomètres) et éloigné, car s'y rendre nécessite un déplacement relativement coûteux en temps de travail (deux heures de trajet aller/retour environ). Chronologiquement, la «retenue» de deux dirigeants de l'usine opère un tournant dans la visibilité médiatique des Molex : elle va faire venir et revenir des journalistes à Villemur. Il ne faudrait pas à propos de cet événement confondre l'effet avec la cause. Si cette retenue va susciter l'intérêt des journalistes (notamment des médias nationaux), elle n'a pas été réalisée dans ce but : il s'agit pour eux d'entrer en contact avec le siège américain de l'entreprise. D'ailleurs ce sont les deux cadres retenus qui préviendront la presse. Ce qui se joue ensuite échappe grandement à la rationalisation sociologique qui voit d'un œil méfiant l'ingénuité des acteurs. Ces «ouvriers» vont en effet largement se contenter d'être ce qu'ils sont avec ce que cela comporte de savoir-vivre et d'humour populaires. Ainsi lorsque les journalistes se rendent sur place dans la nuit, les salariés commencent par leur faire visiter l'usine, leur expliquent leur activité, leur transmettent des documents et leur donnent accès au lieu de la retenue.

On mesure sans doute mal les effets de ce leitmotiv des salariés - le fait qu'ils «n'ont rien à cacher» - sur des journalistes qui ont souvent affaire à des professionnels de la communication dont ils soupçonnent qu'ils « ont quelque chose à leur vendre », qu'ils essaient de les «mener en bateau» (Sobieraj, 2011). En l'occurrence, on va le voir, la plus grande rationalisation des relations presse de la direction va se révéler contre-productive quant à l'image qu'elle renverra des pratiques de l'entreprise.

Une ambiance «Ricard et Front populaire », les intérêts journalistiques à la mise en scène d'un ethos ouvrier

L'empathie manifestée par certains journalistes à l'égard de ce conflit du travail a aussi à voir avec ce qu'est Villemur et ce que sont les protagonistes du conflit. Le bon accueil et la cordialité des Molex, que nous avons pu expérimenter nous-mêmes en situation d'enquête, peuvent, dans l'informalité des rapports qui se nouent inévitablement entre les journalistes et leurs "sources", avoir contribué à la construction du point de vue journalistique sur ce conflit du travail. Le relatif isolement de ce petit bourg industriel où les lieux de sociabilité sont peu nombreux et «les Molex» partout a contribué au resserrement des liens entre journalistes et salariés. Le piquet de grève qui s'installe à l'été 2009 constituera un lieu d'échanges informels extrêmement denses entre journalistes et salariés : on s'y restaure, on y joue aux cartes, aux boules, on y lit les informations en groupe, etc. 
Et donc il y a cette impression physique de l'usine, et il y a aussi les gens qui sont là, quelle impression ils vous ont donnée?

Il y a des images qui s'imposent, alors parfois il faut se méfier des images, mais... bon... là le souvenir que j'en ai, c'est l'été, c'est le barnum, il fait une chaleur pas possible, les gars sont en blouses bleues, et puis on a l'impression que c'est 36 après 36 , il y a une petite ambiance Ricard et Front populaire, avec une telle chaleur qu'on ne sait même pas où se planquer, donc on se met tous à l'ombre du barnum, où il fait chaud, soit en face où il y a un boulodrome. (Entretien avec un correspondant de la PQN, 11 mars 2013)

Plus largement, la démesure entre ce petit bourg industriel (un peu plus de 5000 habitants fin 2009) ${ }^{21}$ et les décisions d'un groupe mondial dont le siège social se trouve à Chicago vient donner corps à l'image qui va durablement résumer le conflit : celui du petit village d'irréductibles résistants au capitalisme globalisé.

Et chez Airbus ça n'existe pas, et chez Sanofi, n’en parlons pas [...] Bref, ça a subsisté cette histoire, parce que c'était isolé, c'était une entreprise au milieu de la campagne, qui continuait à vivre comme si la télé n'existait pas, qui perpétuait cette culture de la classe ouvrière en n'étant confrontée à aucune autre culture dominante. Il n'y avait pas les facs à côté, il n'y avait pas les ingénieurs d'Aérospatiale, c'était un peu comme un village gaulois de la classe ouvrière. (Entretien avec un correspondant de la PQN)

Dès lors, il faut se demander si les journalistes qui viennent couvrir le conflit ne trouvent pas à Villemur de quoi illustrer « la classe ouvrière » telle qu'ils se la représentent ou souhaitent se la représenter. Àl'inverse, la direction de l'usine illustre à merveille la figure du «mauvais patron ».

\section{"Ce sont vraiment des salopards » : la contribution involontaire des dirigeants de Molex à la médiatisation du conflit}

Comparés à des salariés disponibles et disposés à s'exprimer dans les médias, les membres de la direction tentent de maitriser leur communication en choisissant les journalistes auxquels ils souhaitent s'adresser, écartant systématiquement les demandes des autres. Ce «stratégisme » des membres de la direction déplaît particulièrement aux journalistes. À la spontanéité des salariés s'oppose le calcul des membres de la direction. Lorsque ces derniers prennent la parole, ils contribuent involontairement à légitimer la lutte des salariés.

On s'est rendu compte que contre une puissance telle et qui vient d'un pays comme les États-Unis qui est loin de nous, il n'y a pas grand-chose à faire. J'ai un souvenir du patron du site à Molex [...] me dire agacé, puisque je le harcelais

21. Source : recensement INSEE de la commune de 2009. 
de questions sur ces événements, il me dit «vous ne savez pas, monsieur, je vais vous dire... [...] Vous savez, moi j'en ai fermé des boites, et jamais j'ai eu autant de mal qu'en France » [...] Il me dit « tout se passe très bien, on fait un chèque, on s'en va, et voilà », et ça, c'était un des points marquants et j'allais dire choquants. Alors quand on est journaliste, il y a une grosse difficulté, c'est qu'il faut s'impliquer, mais en même temps pas trop, parce que ça joue sur la déontologie et la ligne éditoriale, donc il faut équilibrer tout ça. Et malgré tout, moi il y a eu des moments où je me suis dit «en face ce sont vraiment des "salopards" ». (Entretien avec un journaliste de la PQR, 23 janvier 2013) 22

Un dernier point vient renforcer l'inadaptation des dirigeants étasuniens aux journalistes français : l'inanité de leurs déclarations. En effet, les dirigeants et leurs représentants multiplient les remarques maladroites dans la presse. Dans Le Parisien du 23 avril $2009^{23}$, Lazare Gappeio, incrédule justement quant à leur succès médiatique, fustige par exemple la faiblesse du «niveau intellectuel» de certains salariés. Le 4 mai 2009 dans l'émission «Complément d'enquête » sur France 2, il réitère sa comparaison entre la France et la Somalie ${ }^{24}$ : "On se retrouve à ce niveau comme dans des pays comme la Somalie ou des pays du tiers-monde où on séquestre des gens pour avoir des rançons. Et la rançon qui avait été demandée pour ma libération était de 100 millions d'euros pour vous montrer... C'est pas raisonnable». Enfin, en novembre 2010, Jean-Philippe Lambert, avocat parisien de la direction de Molex, est invité par Audrey Pulvar à la matinale de France Inter et déclare 25 : «Ce qu'il faut juste comprendre, c'est que la direction du groupe Molex a été parfaitement exaspérée par ce que j'appelle le folklore du droit social français. On demande des indemnités absolument mirobolantes puisqu'il faut bien comprendre qu'on demande actuellement à peu près dix ans de salaire ». Comparées aux attitudes des salariés de Molex, celles des dirigeants contribuent ainsi à produire une image négative d'eux.

\section{Des ouvriers qui devancent les attentes des journalistes}

Tout laisse à penser qu'à Villemur-sur-Tarn, les salariés vont au-delà des attentes des journalistes à leur égard en se montrant particulièrement efficaces, disponibles, attentifs et drôles. Ainsi, afin de permettre aux deux porteparoles des salariés de se consacrer, entre autres, aux relations extrêmement chronophages avec les journalistes, ce sont d'autres salariés mobilisés qui

22. Entretien réalisé par O. Baisnée.

23. 〈http://www.leparisien.fr/automobile/constructeurs/molex-les-deux-patrons-sequestresracontent-23-04-2009-488634.php.> (consulté le 23 juin 2017).

24. Repris également dans L'Humanité du 12 août 2009 〈http://www.humanite.fr/node/421945〉 (consulté le 23 juin 2017)

25. Repris par <http://www.plumedepresse.net/lavocat-de-molex-le-folklore-du-droit-socialfrancais/> (consulté le 23 juin 2017). 
prennent en charge la logistique de la lutte, y compris celle qui concerne l'accueil des journalistes. Ce travail de l'ombre est loin d'être latéral et constitue le paratexte du conflit, venant en préparer la lecture journalistique. Ils mangent ainsi régulièrement avec eux. Ces moments d'échanges informels sont propices au développement d'une forte empathie pour le sort de ces salariés «campant» devant leur usine et confrontés à une direction absente.

Ce qui fait la force principale de la mobilisation médiatique des salariés licenciés est aussi leur capacité inattendue à jouer des symboles. Ils font ainsi valoir une identité occitane : "Volem viure é trabalhar al païs a Vilomu » ("Nous voulons vivre et travailler au pays de Villemur») et "Molex Vilomu deu vivre» («Molex Villemur doit vivre») figurent à côté de la croix occitane, alors que certains salariés se déguisent en chevaliers cathares. Mais l'image ${ }^{26}$ qui va rapidement (dès le début de l'année 2009) s’imposer et transformer «les Molex» en mascottes de tous les défilés à Toulouse est celle du village gaulois inventé par René Goscinny et Albert Uderzo.

La première trace que l'on en trouve dans les archives photo constituées en ligne par les salariés 27 remonte à une manifestation à Toulouse le 26 novembre 2008, un mois après le début du conflit sous la forme d'une pancarte sur laquelle est inscrit : «Villemur : le village gaulois qui résiste au prédateur Molex made in USA». Très rapidement les salariés vont reprendre et généraliser la référence : déguisements lors de manifestations et édition d'un t-shirt s'inspirant de la première page de tous les albums d'Astérix. Comme le dira un journaliste de télévision régionale : «Et puis y’a eu ce truc-là aussi. Je veux dire le coup du gaulois, c'était bien vu [...] Ça, ça devait être un truc de journaliste de presse écrite qui a utilisé ce raccourci pour dire “c'est le petit village des irréductibles qui se...”. Un journaliste a dû écrire ça en effet. Et après ils l'ont repris à leur compte».

Le fait que des journalistes soient persuadés que les salariés en lutte se sont inspirés ${ }^{28}$, dans leur mise en scène, d'articles de presse constitue un excellent indicateur de la parfaite adéquation de cette «histoire » avec leurs besoins professionnels. En effet, on sait que les questions d'emploi (et de chômage) (Duval, 2004) sont traditionnellement réputées difficiles à illustrer, particulièrement à la télévision. Comme pour d'autres actualités (international, éducation, etc.), la difficulté à illustrer ces sujets a des effets importants : sujets courts, dont personne ne veut dans les rédactions, relégués en fin de journal, sous-traités. On peut concevoir que l'histoire « des Molex» ait pu provoquer un effet d'aubaine pour évoquer les conséquences de la mondialisation du capitalisme sur l’industrie française.

26. Très rapidement le visuel se substituera aux mots qui deviendront inutiles.

27. Molex, le créateur de chômeurs «http://molex.unblog.fr/photos-de-la-journee-villemur-villemorte-du-6-novembre-2008/> (consulté le 23 juin 2017).

28. Elle est venue, selon un des enquêtés, d'une discussion entre collègues au réfectoire de l'usine. 


\section{Conclusion : contre la tentation stratégiste/intentionnaliste}

La médiatisation de ce conflit du travail déjoue un certain nombre d'attendus sociologiques à propos des mobilisations médiatiques. La capacité à forger et à manier les armes symboliques est traditionnellement repérable auprès de groupes culturellement et scolairement bien dotés et familiers du travail journalistique mieux à même de mobiliser les symboles à défaut de mobiliser le nombre. La tentation peut être grande de mobiliser à l'endroit de ces ouvriers des analyses et des théories formulées pour comprendre les modes d'action collective repérés dans d'autres strates de l'espace social. En l'occurrence, il est bien difficile de déceler quoi que ce soit qui s'apparente aux stratégies de groupes tels qu'Act-Up (Marchetti, 1998), Greenpeace (Baisnée, 2001; Derville, 1997) ou, plus récemment, les Femen. Avec le conflit Molex, le chercheur est confronté à des dominés qui ont bénéficié d'une intense couverture journalistique, positive qui plus est. Comme on a essayé de le montrer, comprendre la félicité médiatique du conflit Molex à l'aune de «stratégies » de communication (avec ce que cela comporte d'intentionnalité, de rationalisation et de savoirfaire), c'est sans doute passer à côté de l'essentiel, mais c'est aussi faire dire à cet objet bien autre chose que ce qu'il a à dire.

S'il y eut bien chez les leaders du mouvement (mais aussi chez leurs avocats) une volonté de faire en sorte que leur lutte soit médiatisée (pour ne pas «crever en silence»), ce succès doit aussi, et peut-être surtout, à des dispositions et des dispositifs mis en œuvre et activés sans être pensés comme devant servir à la médiatisation du conflit. Des savoir-faire pratiques (organiser une restauration collective, trouver des solutions logistiques aux problèmes survenant) et la mise en place d'une répartition efficace du travail militant (et notamment des fonctions de porte-parolat) vont, dans le contexte des rapports entretenus avec les journalistes, contribuer à la popularité journalistique du mouvement et à son cadrage interprétatif. Ainsi, si les journalistes vont avoir tendance à souligner l'unité syndicale du mouvement, la solidarité d'un village envers ses salariés, mais aussi celle qui unissait les salariés, c'est d'abord et avant tout parce qu'ils vont l'expérimenter eux-mêmes dans le cadre de la sociabilité qui se développera aux abords du campement installé aux portes de l'usine ${ }^{29}$.

29. Et qui ne résume pas l'ensemble des situations vécues. Les positionnements critiques à l'égard de la stratégie syndicale ou les stratégies d' « exit » ou plus simplement de retrait se trouvant de fait invisibilisés dans le cadre du piquet de grève. 


\section{Références}

BAISNÉE Olivier, 2001, «Publiciser le risque nucléaire : la polémique autour de la conduite de rejets en mer de l'usine de La Hague », Politix, n054, p.157-181.

Benford Robert D., Snow David A., PlouchaRd Nathalie Miriam (trad.), 2012, «Processus de cadrage et mouvement sociaux : présentation et bilan », Politix, nº 99, p. 217-255.

ChAmPAGNe Patrick éd., 2000, «Le Journalisme et l'économie », Actes de la recherche en sciences sociales, $\mathrm{n}^{0} 131-132$.

Collectif du 9 août, 2017, Quand ils ont fermé l'usine, Paris, Agone.

DERVILLE Grégory, 1997, «Le combat singulier Greenpeace-SIRPA. La compétition pour l'accès aux médias lors de la reprise des essais nucléaires français ", Revue française de science politique, vol.47, nº 5, p. 589-629.

DuvalJulien, 2004, Critique de la raison journalistique. Les transformations de la presse économique en France, Paris, Éd. du Seuil.

FILLIEULE Olivier, 2007, «On n'y voit rien. Le recours aux sources de presse pour l'analyse des mobilisations protestataires ", L'Atelier du politiste. Théories, actions représentations, P. Favre, O. Fillieule et F. Jobard éd., Paris, La Découverte, Paris, p. 215-240.

-, 1996, "Archives policières, sources de presse et manifestations de rue », Les Cahiers de la sécurité intérieure, n²6, octobre, p. 153-176.

GoffmAn Erving, 1974, Frame Analysis: An Essay on the Organization of the Experience, New York, Harper.

JOUANNEAU Solène, 2012, «Pour une lecture française plus ethnographique de la frame analysis», Genèses, n88, p.132-134.

MARCHETTI Dominique, 1998, "Les conditions de réussite d'une mobilisation médiatique et ses limites : l'exemple d'Act Up », La Politique ailleurs, Centre universitaire de recherche sur l'action publique et le politique : épistémologie et sciences sociales (CURAPP) éd., Paris, PUF, p. 277-297.

MISCH I Julian et Renahy Nicolas, 2008, «Pour une sociologie des mondes ruraux», Politix, n०83, p. 9-21.

Peterson Richard A., 1992, "La fabrication de l'authenticité [La country music] », Actes de la recherche en sciences sociales, nº 93, p. 3-20.

REINERT MaX, 1983, "Une méthode de classification descendante hiérarchique : Application à l'analyse lexicale par contexte ", Cahiers de l'analyse des données, n³, p.187-198. Sobieraj Sarah, 2011, Soundbitten, The Perils of Media-Centered Political Activism, New York, New York University Press. 